Nucleic-Acid Signatures at Three Levels of Readiness for Biowatch

R. P. Mariella

June 14, 2013 
This document was prepared as an account of work sponsored by an agency of the United States government. Neither the United States government nor Lawrence Livermore National Security, LLC, nor any of their employees makes any warranty, expressed or implied, or assumes any legal liability or responsibility for the accuracy, completeness, or usefulness of any information, apparatus, product, or process disclosed, or represents that its use would not infringe privately owned rights. Reference herein to any specific commercial product, process, or service by trade name, trademark, manufacturer, or otherwise does not necessarily constitute or imply its endorsement, recommendation, or favoring by the United States government or Lawrence Livermore National Security, LLC. The views and opinions of authors expressed herein do not necessarily state or reflect those of the United States government or Lawrence Livermore National Security, LLC, and shall not be used for advertising or product endorsement purposes.

This work performed under the auspices of the U.S. Department of Energy by Lawrence Livermore National Laboratory under Contract DE-AC52-07NA27344. 


\title{
Nucleic-Acid Signatures at Three Levels of Readiness for Biowatch
}

Report by R. Mariella Jr. [see acknowledgement for list of persons with whom Author communicated about this report]

Prepared for Nat'l Acad./Institute of Medicine Workshop, 25-26 June 2013

This work performed under the auspices of the U.S. Department of Energy by Lawrence Livermore National Laboratory under Contract DE-AC52-07NA27344.

\begin{abstract}
Statement of Work for:
Raymond P. Mariella, Jr. - Commissioned Paper

As requested by India Hook-Barnard, RSO

For the IOM Board on Health Sciences Policy Stand Alone Workshop

The Institute of Medicine (IOM) requests that the following commissioned paper be written,

Potential Technologies for BioWatch: State of the Art on Nucleic-acid Signatures. Raymond P. Mariella, Jr. will have the opportunity to present the commissioned paper at the IOM's Strategies for Cost-effective and Flexible Biodetection Systems that Ensure Timely and Accurate Information for Public Health Officials stand alone workshop on June 25-26, 2013. The IOM workshop is sponsored by the Department of Homeland Security's Office of Health Affairs and will explore cost-effective systems that would meet requirements for BioWatch as an automated detection system for aerosolized agents (alternative technologies for autonomous detection).

The paper by Raymond P. Mariella, Jr. will discuss nucleic-acid signatures (PCR, microarrays, and other probe-based systems) at three levels of readiness for BioWatch:

1. Tier 1: fully automated bio-detection system, capable of 24/7/365 unattended outdoor and indoor operation, that will be at a Technology Readiness Level of TRL 6 plus by 2016 .

2. Tier 2: similar requirements but will not reach a TRL 6 plus level until sometime between 2016 and 2020.

3. Tier 3: technologies that have the potential of meeting or exceeding the BioWatch requirements, but a fully automated, TRL 6 plus system would take us beyond the 2020 time frame. For these technologies, describe the current critical paths ('long poles') in meeting the BioWatch requirements and how they might be addressed.
\end{abstract}

\section{Disclaimer:}

This Report, focusing on nucleic-acid-based detection of bioaerosol agents, must be only a subset of a larger plan. This report does not address food-borne, water-borne, vector-borne, or human-to-human transmission of infectious agents.

Author's strong recommendation for both planning and implementation: Since the Committee is examining what would be entailed to provide an autonomous system that performed BioWatch assays and provided CDC/LRN with the same amount of nucleic-acid-sequence information, along with the same reliability and accuracy as the validated laboratory assays, in the Author's opinion, the CDC/LRN and any others who will rely upon such information to make calls of detection of an agent need to be involved in each step in the planning and development of such an autonomous system. 


\section{Caveat: Full, End-to-End System Integration is difficult!}

The Author's general premise is that integration of disparate components into an automated Bioagent Detection and Identification System that attempts to perform the same functions as trained laboratory personnel is extraordinarily difficult numerous, high-quality, COTS instruments exist that perform the functions of the individual steps, yet field tested and proven integrated systems are few. It is a particular challenge in any automated fluidic system to make $100 \%$ reliable fluidic interconnections, along with the necessary methods to transport, meter, and process samples and reagents so that the laboratory-proven assays, that normally use trained personnel with pipettes, filters, and centrifuges, perform just as well in the automated system. Challenges to system integration can arise from something as basic as an output volume of one stage being incompatible with the next stage's input volume, or differing buffer concentrations between one stage and the next, or a "short spin" on the centrifuge being needed in between, etc. One extremely important hurdle for system integration is that a manufacturer may refuse to provide what seems to be a minor modification to a selected component that is needed to permit integration and, if a third party performed the needed modification, the original manufacturer may refuse to service the modified component and declare that the modification voided the warranty. This is particularly likely when the desired component might be projected to have total annual sales of a few dozen or less. Thus, a standalone instrument may work very well in an LRN or BioWatch laboratory and, yet, effectively not be available for system integration.

\section{Table of Contents}

I. Introductory thoughts and overview

II. Performance considerations

III. Nucleic-acid signatures

IV. Assays

a. Digital sample processing and digital PCR

b. An aside on limits of detection

c. Topics for probe-based assays

d. Role of viability/infectivity assays

e. Sequencing by hybridization

f. Direct sequencing of probe regions

V. Instruments, platforms, and problems

a. Polymerase-based amplification and detection

b. Microarrays

c. Unassembled sequencing

VI. Field-tested autonomous systems

a. APDS

b. M-BAND

c. Possible future systems

VII. References 
I. Introductory thoughts [it is useful the keep in mind how and where the nucleicacid signatures are measured]

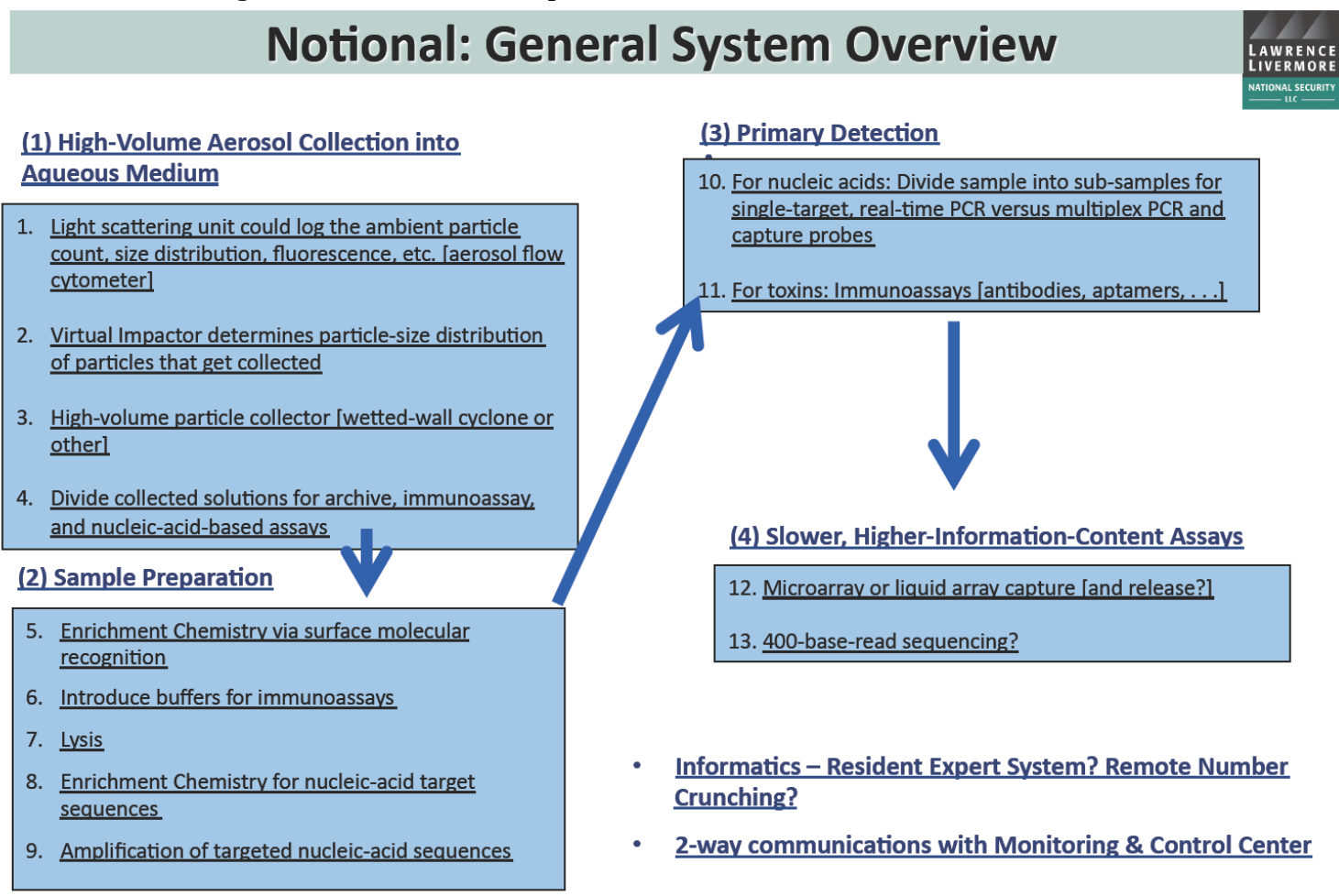

Figure 1. Block diagram of components of a notional next-generation system, including components and functions that may be incorporated in a staged manner: items $2-4,6-11$, are either incorporated or could easily be incorporated in systems that could be deployed by 2016. Item 1, pre-collection particle characterizer, could probably be incorporated as part of a next-generation system by 2016 , but certainly by 2020 , if the cost/benefit analysis favored its incorporation. Item 5, selective capture/enrichment of pathogens via surface molecular recognition is listed, although extensive $R \& D$ would be required to make this a reality ${ }^{1}$. Item 8 is also somewhat of a catchall, covering simple chemistries, such as the Boom capture/release of nucleic acids, but may also include emerging techniques, including even capture, isolation, and release of nucleic-acid regions of interest on either fixed or liquid microarrays ${ }^{2}$. Item 12 would likely require its own multiplex target amplification, prior to hybridization, and incorporating such a process, along with the logistics of replacement of single-use microarrays or the reconditioning of a microarray, along with automated readout of hybridization patterns, seems destined for deployment beyond 2020. Item 13 would perform a very desirable function, but attempting an automated Metagenomic analysis of a sample seems like a VERY "long pole" in the tent. Preceding sequencing with multiplex target amplification may make this automatable by 2020 [the Author awaits the participation of Dr. Jovanovich in our Workshop]. 
II. Performance considerations - Assuming that the areal density of samplers is limited [by costs, among other reasons]

Premise: A release of agent can dissipate and yet remain at physiologically-relevant concentrations, for large areas, putting the public at risk, so the aerosol sampler needs to bring in as many $\mathrm{L} / \mathrm{min}$ as is practical.

Considerations: Without regard to any specific autonomous system or placement of a multitude of such systems throughout an urban setting, let us simply consider what might happen to persons who were exposed for 30 minutes to a release that contained $\mathrm{N}$ viable bacteria/ $\mathrm{m}^{3}$.

From us EPA, Exposure Factors Handbook ${ }^{3}$, chapter 6, adult humans respire, on a 24 -hr average, roughly $10 \mathrm{~L} /$ minute, so 30 minutes $=300 \mathrm{~L}$ breathed. Since $1000 \mathrm{~L}=$ $1 \mathrm{~m}^{3}$, "average" breathing would pull in $0.3 \mathrm{~N}$ viable bacteria over 30 minutes. Depending upon the details of the aerosol, it could be that effectively every viable bacterium breathed in could be retained within the lungs.

The infectious dose of some bacteria and viruses has been measured to be essentially a single viable bacterium or particle with infectious virions ${ }^{4}$. Regarding viruses, from p.91 of Kamps-Hoffman-Preiser5:

Within nasal secretions, millions of virus particles per ml are shed, so that a $0.1 \mu \mathrm{laerosol}$ particle contains more than 100 virus particles. A single HID (human infectious dose) of influenza virus might be between 100 and 1,000 particles.

Although respiration rates amongst the very young or the elderly may be lower, the infectious dose for some agents may be lower, as well. See p2239 of Inglesby ${ }^{6}$ :

Recently published extrapolations from primate data suggest that as few as 1 to 3 spores may be sufficient to cause infection. The dose of spores that caused infection in any of the 11 patients with inhalational anthrax in 2001 could not be estimated although the 2 cases of fatal inhalational anthrax in New York City and Connecticut provoked speculation that the fatal dose, at least in some individuals, may be quite low.

Thus, if $\mathrm{N}$ for a particular agent remained above $10 / \mathrm{m}^{3}$, for example, then a 30 minute exposure could lead to infection of persons in that vicinity, if the infectious dose were very low. The location of an autonomous system may be far enough away that the aerosol concentration of potentially pathogenic bacteria or virus may have dropped to a much lower level, when the release reached it - this report author argues that the father apart one wishes to locate such autonomous systems, the higher each system's volumetric sampling and sensitivity need be to avoid missing an attack. 
Regarding background bioaerosols, as reported by Jensen in his report on Atmospheric Background Aerosols ${ }^{8}$ [see page 9 of his report]:

(U) Time-resolved concentration profiles of bacteria and spores showed that spore concentrations of 11,000 per cubic meter $\left(m^{\wedge} 3\right)$ occurred and that the concentration curve contained many "bumps" resembling an aerosol cloud passage, as well as many short duration spikes.

Also of relevance for assays that do not distinguish "live/dead," such as PCR and most other nucleic-acid-sequence-based assays,

... there were approximately 50 total airborne bacteria for every culturable bacterium.

All of this is to say that it is not hard to envision a situation in which a potentially pathogenic bacteria or virus could be present at physiologically relevant concentrations, while remaining well below the background concentration of physically similar, non-pathogenic aerosolized bacteria and viruses.

\section{Nucleic-acid signatures}

What comprises nucleic-acid signatures? Should an autonomous system include genomic, plasmid, and even rRNA in bacteria by 2020? Informatics - what level of sequence ID is required to call a positive? How well conserved are "Consensus Sequences"? How much deviation is acceptable? [especially tricky for singlestranded DNA and RNA viruses] Experimentally, what level of sequence mis-match or incorrect base calling is acceptable?

How do signatures need to change as strains evolve or new species \& sub-species are discovered ${ }^{9-11}$, both within the species of the pathogen and with its near neighbors?

The author expects that such discussions of nucleic-acid signatures and their evolution over time will proceed within the Workshop Panels "BioWatch Information for Decision Making" and "State of the Art on Genomic Sequencing," however, this author wishes to convey, anecdotally, that it is his understanding that for at least one species of pathogenic virus there are no conserved sequences longer than 14 bases, making a single-target assay problematic for that agent. Moreover, for single-stranded DNA and RNA viruses, concepts such as "Quasi-species" are often needed and appropriate ${ }^{12,13}$. 


\section{Assays}

LRN and BioWatch laboratory assays, today, are certainly performed with commercial instruments, but, due to the considerations at the beginning of this report on the challenges of system integration, we will not compare various commercial, standalone components or instruments, specifically. However, the author hopes what follows will be discussion-provoking -

\section{a. Digital sample processing and digital PCR}

Given the rigor and time-consuming nature of creating fully tested and validated assays within the guidance of Public-Health-Actionable Assays*, the following is likely NOT fieldable by 2016, but could possibly be TRL6 by 2020: digital PCR ${ }^{14}$

Gevensleben detected and quantified circulating free plasma DNA with [dropletdigital PCR] ddPCR ${ }^{15}$; Strain did HIV quantification ${ }^{16}$ as did Henrich ${ }^{17}$. Kelley, et al., reported sensitive quantification of Methicillin-Resistant Staph. Aureus using digital $\mathrm{PCR}^{18}$. Roberts described a digital PCR diagnostic assay for ocular Chlamydia trachomatis ${ }^{19}$ : unlike other nucleic acid amplification tests it "... requires no external or internal calibration yet delivers a highly accurate estimation of target load."

Morisset ${ }^{20}$ - on quantification:

"The excellent performance of the tested parameters enables the quantification of samples from different matrices, using DNA extracted with common methods without up-front DNA quantity estimation," ... "The limits of quantification, trueness, and repeatability of the duplex assay comply with international recommendations." And ... "ddPCR running costs are lower than those of standard qPCR technology, given the superior throughput... " There are numerous other publications that used

\footnotetext{
* "Framework for a Biothreat Field Response Mission Capability" Developed by an Interagency Working Group (IAWG) convened to develop guidance to First Responders for the Biological Assessment of Suspicious Powders

Bert Coursey [DHS, S\&T] and Richard B. Kellogg [LRN, CDC]
}

Public Health Actionable Assays (PHAAs) are laboratory-based assays that are used to support public health decisions and which have been qualified according to consensus performance standards developed by a recognized and representative body from the stakeholder community'. PHAAs are developed and utilized t o support public health act ions involving the potential exposure of an individual or, more commonly, groups of individuals to biothreat materials such as $B a$ spores. PHAAs have high specificity, high sensitivity, and are highly robust to provide critical information on agent -specific confirmation and further characterization to support public health decisions such as initiating a national or local health alert warning, initiating a public health investigation, conducting risk assessments to support post exposure prophylaxis distribution and initiating public health risk communications. These assays are intended to be employed in well-established controlled laboratory environments, such as an LRN reference laboratory, using an established ConOps and where professional training and user proficiency certification are established. Connected with Stakeholder Panel on Agent-Detection Assays [SPADA] 
a variety of platforms ${ }^{21-25}$. Exactly how a system integrator would implement digital PCR, even by 2020, is an open question, but worth considering.

\section{b. An aside on limits of detection ${ }^{\dagger}$}

Apropos of the previous discussion of "digital PCR," but without diving into what is famously known as "Receiver Operating Characteristic" curves, let us, briefly, review what is meant by a "false negative." It has been demonstrated, for almost as long as PCR has been used, that an optimized PCR assay can perform down to the Poisson limit of detection ${ }^{26}$. That is, if the PCR reaction solution has no inhibitors, but does have the appropriate primers, master mix, and only a single copy of the target DNA, a positive detection signal can be observed ${ }^{26}$. Without referring to any particular autonomous instrument, if it collects $1 \mathrm{~m}^{3} / \mathrm{min}$ and has a $10 \%$ overall processing throughput from collection to PCR reaction, including archiving/dividing some of the collected sample, then if a particular bacterium enters the collector as an aerosol with concentration of $10 / \mathrm{m}^{3}$ and the system collects air for 1 minute, then on average the PCR reaction will have one bacteria target in its reaction volume. However, assuming that Poisson statistics apply, $1 / \mathrm{e}=37 \%$ of the time there will be NO target in the PCR reaction and one can, therefore, expect to "miss" the detection of this aerosolized bacteria at this aerosol concentration, $37 \%$ of the time. If there were three separate PCR chambers each with $10 \%$ overall throughput from collector to it, each with a different sequence signature from the bacterium, for example, then the bacterium would miss all three reactions only $1 / \mathrm{e}^{3}=5 \%$ of the time. If the manufacturer claimed a limit of detection with all three signatures positive for this bacterium of $10 / \mathrm{m}^{3}$ in 1 minute, then the system would fail to give this positive $37 \%$ of the time. If only one or two signatures came up positive, the result could be termed "indeterminate," for example, with its own particular concept of operation.

Note: if a multiplex assay with three sequence signatures per target organism also obeyed Poisson statistics and was incorporated in a similar autonomous system that had $30 \%$ throughput from collector to reaction chamber, due to less division of the collected sample, then it would produce a false negative $5 \%$ of the time for this concentration of aerosol.

\footnotetext{
† This Author understands that a careful study was made of BioWatch assays, implemented under both single-plex and multiplex formats, but, as of this writing, he has not been able to read any resulting report. Such a study would bear upon the discussion about limits of detection and Poisson statistics, immediately above.
} 


\section{c. Topics for probe-based assays}

What accuracy, time to perform, cost...?

Sensitivity, specificity: require $(+)$ call for all or only some physiologically-relevant concentrations? No false $(+)$ from non-virulent subspecies

Time to perform assay ${ }^{27}$ : "Using Thermococcus kodakaraensis polymerase ... we obtained an accurate, 35 cycle amplification of an 85-base pair fragment of E. coli 0157:H7 Shiga toxin gene in as little as $94.1 \mathrm{s."}$ It seems unlikely that the time to perform the polymerase-based amplification will be the limiting factor on processing time, even with slower thermal cyclers.

Difficulty to perform manually and/or automatically What level of sample preparation is required?

Ability to transfer Informatics, reagents to other locations

Cost of startup for each detection system, consumables, instrument maintenance, Ability to include (+) and (-) controls

Expandability - MPX versus single-plex

What protocol/procedures are required for validation [PHAA]? [time, \# of assays to run, experimental accuracy ...]

\section{Require at least two, simultaneous or sequential hybridization events, per signature region, with at least three signatures required per target bacterium or virus?}

This Author, in contacting numerous practitioners, has found divergent positions on the topic of which assays to incorporate into autonomous detection systems. A number of skilled laboratorians endorse the BioPlex ${ }^{\circledR}$ assay, based on multiplex PCR amplification and subsequent hybridization to a liquid array of bead types. A different group of skilled laboratorians endorse real-time PCR with Taqman ${ }^{\circledR}$ or Molecular Beacon ${ }^{\circledR}$ probes. The BioPlex ${ }^{\circledR}$ assay was fielded for the DHS in the Northrop-Grumman APDS [see below], with seemingly no false positives or false negatives. The real-time PCR was tested in the Hamilton-Sundstrand M-BAND in realistic settings with good results [see below]. Given the rushed nature of the preparation of this report and the relative lack of overwhelming data, no specific recommendation is made in this report, other than to recommend detailing the time and trouble, within the PHAA guidelines [see previous footnote ${ }^{*}$ ], to produce and validate each approach, both as individual signatures evolve and as the number of target bacteria and viruses increases. Will (+) and (-) controls be required for each reaction volume?

d. Role of viability/infectivity assays? Part of a long-term system design could include local viability assays, triggered by initial positive ID with nucleic-acid signature. Rapid viability assays have been developed that can even be applied to slow-germinating Bacillus endospores ${ }^{28-30}$, but would require profound changes 
in automation, since actual culture is required. For some endospores, an accelerated method can still require 12 to 24 hours for accurate results ${ }^{30}$. Incorporation would require an analysis of the tradeoffs between dispatching a courier to retrieve a positive sample for viability and other testing at a staffed laboratory versus attempting to incorporate such an assay in a standalone system, or some combination of both.

\section{e. Sequencing by hybridization [SBH] ${ }^{31-33}$ to planar array.}

The Author sees SBH as having major obstacles to incorporation into a standalone, autonomous system, even by 2020. Topics such as probe length and stringency of hybridization specificity, Informatics/pattern-recognition imaging equipment and software, as well as tradeoffs between copy number, size of array, and time to equilibrate, can probably be addressed by 2020, - but can sample prep be automated and what sort of consumables supply and robotic handling of chips for performing assays? Would the system re-use chips, or sequentially use minichips on a larger chip with complicated fluidics? What accuracy results from hybridizing with a complex environmental mixture? To what extent could selective MPX amplification of regions of interest knock down confounding sequences and non-specific binding?

To this Author, the "long poles" for this application are establishing and automating the sample preparation that is needed, prior to hybridization, as well as robotic chip handling and necessary fluidics for introducing new chips and enabling equilibrated hybridizations [well-defined stringency of hybridizations]. Overall cost/performance would also need to be considered.

f. Direct sequencing of probe regions via pyrosequencing, single-molecule sequencing, etc - no assembly [what constitutes an "Informative Region" of genome, plasmid [bacteria], virus?

Although Next-Generation Sequencing continues to increase the power and affordability of sequencing a human's individual's genome, how well do these increases enhance rapid, autonomous sequencing of pathogenic bacteria and viruses from complex environmental samples?

\section{Issues of sample preparation for direct sequencing}

Starting from a raw aerosol sample [captured in a wetted-wall particle collector?], how many years will it be before the DHS can field a fully-automated system that performs:

1. Possible selective capture of virulent bacteria and viruses [enrichment of pathogens out of a mixture of innocuous microbes and human detritus] using a surfacerecognition capture, possibly with derivatized magnetic beads;

2. Spore and cell lysis;

3. Extraction and purification of nucleic acids;

4. Possible enrichment/capture of nucleic-acid sequences that are signatures of 
virulence in human pathogens;

5. Whatever amplification is necessary [priming off of the virulence regions?];

6. Fragmentation and size selection, as needed;

7. Addition of bar codes, as needed;

8. Ligation, as needed;

9. Adjustment of buffers, washing, separation, as needed for every step to produce the necessary sequencing library;

10. Sequencing with each read being $\approx 400$ bases;

11. Maybe quantification of starting copy number;

12. Informatics analysis of sequence, including "identification" of strain and sub-strain variants [particularly tricky for ss-DNA or RNA viruses] resulting in actionable output.

\section{Instruments, platforms, and problems}

Polymerase-based assays that use thermal cyclers - if disposable, single-use tubes or wells, what burden of consumables is incurred? If flow-through, what difficulty in cleaning out all prior material? Lifetime/aging of the flow-through, thermalcycling chamber/tubing [repeated exposure to bleach]?

If fixed-surface [Microarray?] is used, how to integrate flow system and hybridization chamber - swish the sample back and forth or use diffusion, only? Time for equilibration/signal versus starting copy number and concentration of possibly interfering sequences?

Size and cost of each array?

What sample prep, including either non-specific or specific [i.e, virulence region], multiplexed amplification [similar to AmpliSeq ${ }^{\circledR}$, but targeted to "Regions of Interest"]

Leak-free insertion of new array for next sample - what complexity, reliability of robotics would be required? How about a large microarray with many sub-arrays in one large fluidic manifold, where each microarray is operated, individually? What pattern-recognition software exists or needs to be created?

Distinguish multiple targets, simultaneously?

Reproducibility and quantification?

Would the DHS application require a custom sequencing platform? [existing systems that slowly provide $10^{8}$ or $10^{9}$ reads do not seem to be a good match to the DHS workspace] Also, carryover of reads from prior sequencing runs could prevent simple adoption of an existing sequencing platform.

What choices are there for robotic or microfluidic platforms to perform sample handling and sample preparation for a customized sequencing instrument [IntegenX and others]? 
What software is needed to reduce or eliminate the local need for experienced Informatics personnel to interpret sequence data [natural sequence variability as well as instrumental mis-calls]? If resident, expert software is feasible, what is its time frame to reach TRL 6? It is this Author's understanding that multiple groups are already working on such software, so a system with custom sequencing and resident, expert software could be available in the 2016 - 2020 timeframe.

Performance of both microarray platforms and sequencing platforms would likely benefit significantly from targeted multiplex polymerase amplification of regions of interest. There would be less confounding data and chemistry in the readout step and the pattern-recognition or base-calling software would have an easier job.

\section{Field-tested autonomous systems}

Mature candidates -

TRL9 - Northrop-Grumman Autonomous Pathogen Detector System [APDS] performed autonomous operation, using multiplexed PCR assays, for the DHS for some period with no false $(+)$ or $(-)$. General operation has been described by Regan, et al. ${ }^{34}$.
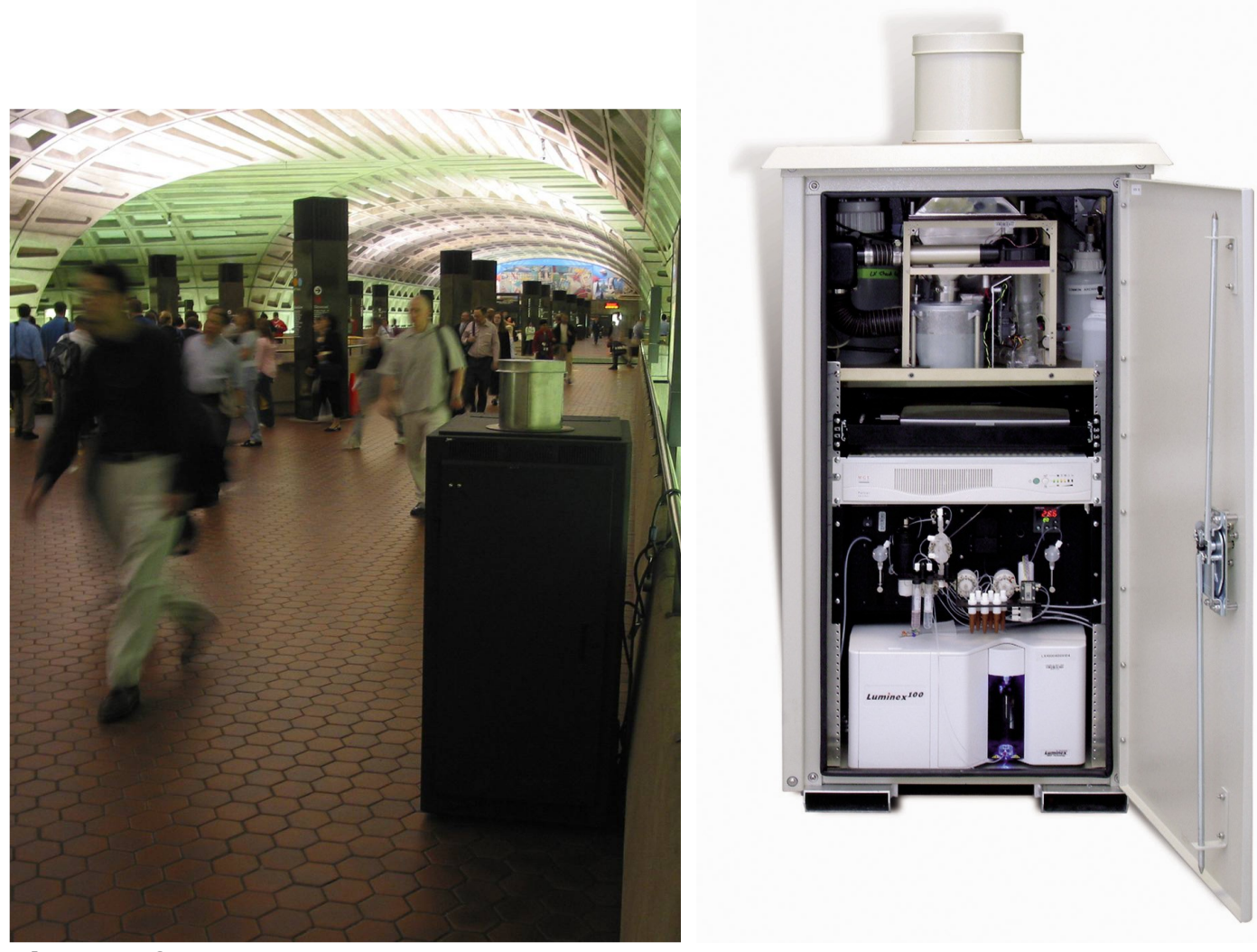

Photos of APDS 
TRL8 - Hamilton-Sundstrand Microfluidic-Bioagent Autonomous Networked Detector [M-Band] PositiveID has successfully detected and identified blind samples provided by the DHS, as described by Sanchez, et al. ${ }^{35}$.
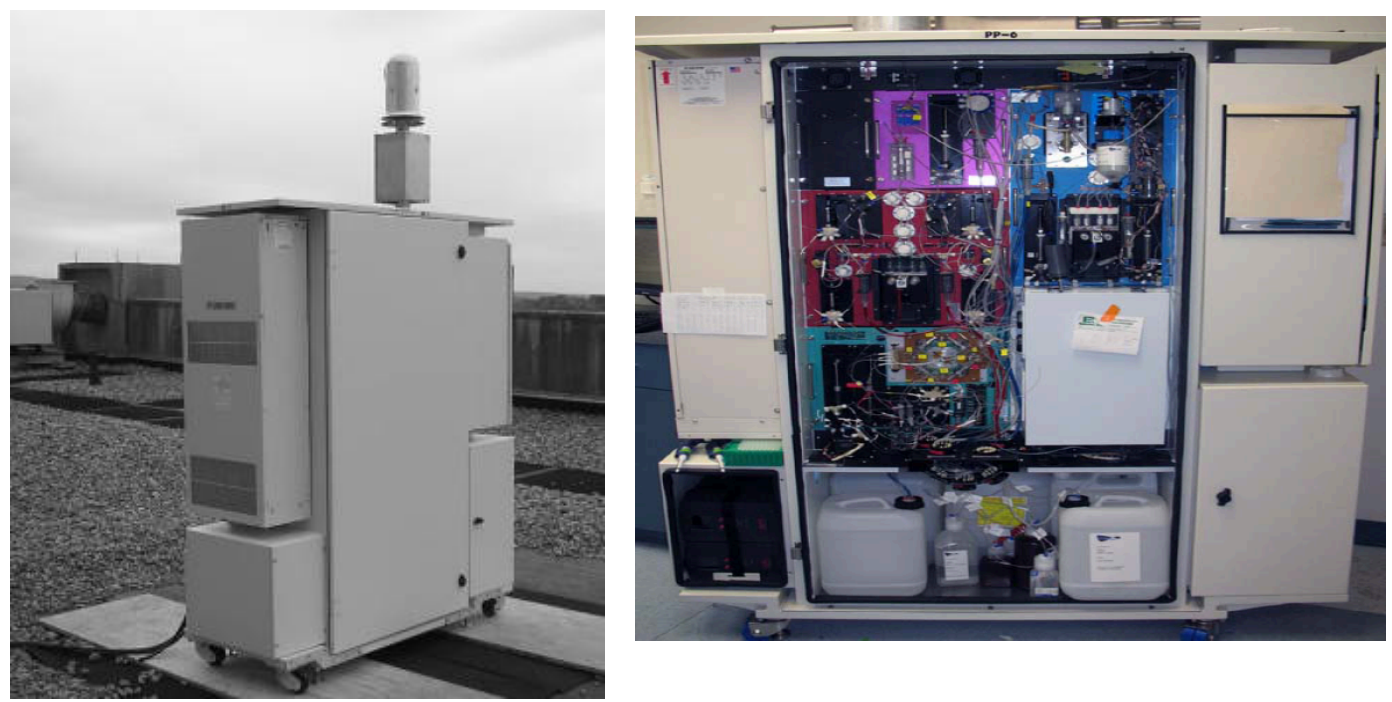

Photos of M-BAND

Both the Northrop-Grumman system, which collects $1.7 \mathrm{~m}^{3} / \mathrm{min}$, and the HamiltonSundstrand system, which collects $0.4 \mathrm{~m}^{3} / \mathrm{min}$, are well beyond the TRL6 point for field deployment by 2016, assuming no fundamental changes in their assays.

The Northrop-Grumman system appears to have an easier path forward to expansion of number of assays [the liquid array approach has a built-in capability to handle up to 100 target sequences] and runs internal $(+)$ and $(-)$ controls for every polymerase-amplification reaction. 
Due to demand for inexpensive sequencing of individual human genomes, some relevant science and technology continue to advance - not an integrated, fullyautomated stand-alone system, but good long-term prospects, one example: IntegenX - automated sample preparation for sequencing [work performed for DTRA]

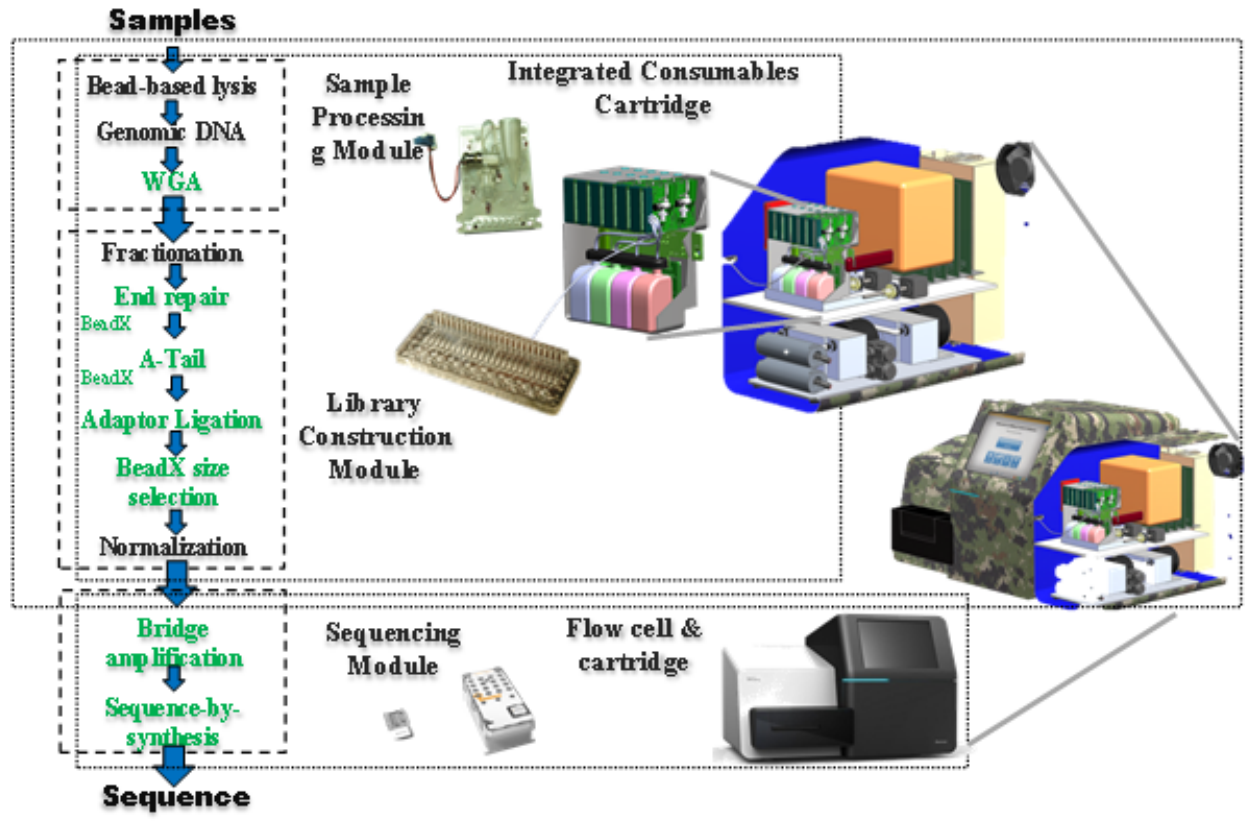

\section{Acknowledgement}

The Author gratefully acknowledges discussions with:

Thomas Bunt [LLNL]

Shea Gardner [LLNL]

Staci Kane [LLNL]

Pejman Naraghi-Arani [LLNL]

David Rakestraw [LLNL]

Marilyn Ramsey [LLNL]

Tom Slezak [LLNL]

Mark Wagner [LLNL]

Lewis Wogan [LLNL]

John Dzenitis [BioRad]

Phillip Belgrader [BioRad]

M. Allen Northup [formerly of Cepheid and MFSI] 
David Walt [Tufts University

Stevan Jovanovich [IntegenX]

Kimothy Smith [PositiveID/MFSI]

Lyle Probst [PositiveID/MFSI]

George Dizikes [Illinois Department of Public Health]

Brent Chyna [State of Illinois, Astrix Technology Group, Inc]

Bernadette Johnson [Lincoln Labs]

Cynthia Bruckner-Lea [PNNL]

Michael Farrell [CDC]

Darren Link [Raindance] 
References

1. Pratt, E.D., Huang, C., Hawkins, B.G., Gleghorn, J.P. \& Kirby, B.J. Rare cell capture in microfluidic devices. Chemical Engineering Science 66, 1508-1522 (2011).

2. DuBose, A.J., et al. Use of microarray hybrid capture and next-generation sequencing to identify the anatomy of a transgene. Nucleic Acids Res. 41(2013).

3. Moya, J. \& al., e. Exposure Factors Handbook, (U.S. Environmental Protection Agency (EPA). National Center for Environmental Assessment, Washington, DC; EPA/600/R-09/052F., 2011).

4. Jones, R.M., Nicas, M., Hubbard, A., Sylvester, M.D. \& Reingold, A. The Infectious Dose of Francisella tularensis (Tularemia). Applied Biosafety 10, 227-239 (2005).

5. Kamps, B.S., Hoffmann, C. \& Preiser, W. Influenza Report 2006 - a medical textbook, (Flying Publisher, 2006).

6. Inglesby, T.V. Anthrax as a biological weapon, 2002: Updated recommendations for management (vol 287, pg 2236, 2002). Journal of the American Medical Association 288, 1849-1849 (2002).

7. Peters, C.J. \& Hartley, D.M. Anthrax inhalation and lethal human infection. The Lancet 359, 710-711 (2002).

8. Jensen, J.G. Effect of Atmospheric Background Aerosols on Biological Agent Detectors. (ed. (A3SC), H.U.S.A.F.H.U.D.D.f.C.) 30 (2007).

9. Zeytun, A., et al. Complete Genome Sequence of Francisella philomiragia ATCC 25017. Journal of bacteriology 194, 3266-3266 (2012).

10. Jackson, J., et al. Francisella tularensis Subspecies holarctica, Tasmania, Australia, 2011. Emerging Infectious Diseases 18, 1484-1486 (2012).

11. Signarovitz, A.L., et al. Mucosal Immunization with Live Attenuated Francisella novicida U112 Delta iglB Protects against Pulmonary F-tularensis SCHU S4 in the Fischer 344 Rat Model. PLoS ONE 7(2012).

12. Lauring, A.S. \& Andino, R. Quasispecies Theory and the Behavior of RNA Viruses. PLoS Pathog 6, e1001005 (2010).

13. Volz, E.M., Koelle, K. \& Bedford, T. Viral Phylodynamics. PLoS Comput Biol 9, e1002947 (2013).

14. Vogelstein, B. \& Kinzler, K.W. Digital PCR. Proceedings of the National Academy of Sciences of the United States of America 96, 9236-9241 (1999).

15. Gevensleben, H., et al. Noninvasive Detection of HER2 Amplification with Plasma DNA Digital PCR. Clinical Cancer Research (2013).

16. Strain, M.C., et al. Highly Precise Measurement of HIV DNA by Droplet Digital PCR. PLoS ONE 8, e55943 (2013).

17. Henrich, T.J., Gallien, S., Li, J.Z., Pereyra, F. \& Kuritzkes, D.R. Low-level detection and quantitation of cellular HIV-1 DNA and 2-LTR circles using droplet digital PCR. Journal of virological methods 186, 68-72 (2012). 
18. Kelley, K., Cosman, A., Belgrader, P., Chapman, B. \& Sullivan, D.C. Detection of Methicillin-Resistant Staphylococcus Aureus by A Duplex Droplet Digital Polymerase Chain Reaction. Journal of Clinical Microbiology (2013).

19. Roberts, C.h., et al. Development and evaluation of a next generation digital PCR diagnostic assay for ocular Chlamydia trachomatis infections. Journal of Clinical Microbiology (2013).

20. Morisset, D., Štebih, D., Milavec, M., Gruden, K. \& Žel, J. Quantitative Analysis of Food and Feed Samples with Droplet Digital PCR. PLoS ONE 8, e62583 (2013).

21. Straub, T., et al. Estimated copy number of Bacillus anthracis plasmids pX01 and pXO2 using digital PCR. Journal of Microbiological Methods 92, 9-10 (2013).

22. Ottesen, E.A., Hong, J.W., Quake, S.R. \& Leadbetter, J.R. Microfluidic digital PCR enables multigene analysis of individual environmental bacteria. Science 314, 1464-1467 (2006).

23. Tadmor, A.D., Ottesen, E.A., Leadbetter, J.R. \& Phillips, R. Probing Individual Environmental Bacteria for Viruses by Using Microfluidic Digital PCR. Science 333, 58-62 (2011).

24. Whale, A.S., et al. Comparison of microfluidic digital PCR and conventional quantitative PCR for measuring copy number variation. Nucleic Acids Res. 40(2012).

25. Shen, F., et al. Multiplexed Quantification of Nucleic Acids with Large Dynamic Range Using Multivolume Digital RT-PCR on a Rotational SlipChip Tested with HIV and Hepatitis C Viral Load. Journal of the American Chemical Society 133, 17705-17712 (2011).

26. Sykes, P.J., et al. QUANTITATION OF TARGETS FOR PCR BY USE OF LIMITING DILUTION. Biotechniques 13, 444-449 (1992).

27. Maltezos, G., et al. Exploring the limits of ultrafast polymerase chain reaction using liquid for thermal heat exchange: A proof of principle. Applied Physics Letters 97(2010).

28. Kane, S., et al. Application of a high throughput rapid viability polymerase chain reaction (RV-PCR) method for detection of Bacillus anthracis and its surrogates. Abstracts of the General Meeting of the American Society for Microbiology 106, 627-627 (2006).

29. Letant, S.E., et al. Most-Probable-Number Rapid Viability PCR method to detect viable spores of Bacillus anthracis in swab samples. Journal of Microbiological Methods 81, 200-202 (2010).

30. Letant, S.E., et al. Rapid-Viability PCR Method for Detection of Live, Virulent Bacillus anthracis in Environmental Samples. Applied and Environmental Microbiology 77, 6570-6578 (2011).

31. Drmanac, R., Labat, I., Brukner, I. \& Crkvenjakov, R. SEQUENCING OF MEGABASE PLUS DNA BY HYBRIDIZATION - THEORY OF THE METHOD. Genomics 4, 114-128 (1989).

32. Palacios, G., et al. Panmicrobial oligonucleotide array for diagnosis of infectious diseases. Emerg Infect Dis 13, 73-81 (2007). 
33. Thissen, J., Gardner, S., McLoughlin, K., Slezak, T. \& Jaing, C. Rapid analysis of known and unknown pathogens using a pan-microbial detection microarray. Int. J. Infect. Dis. 14, E272-E272 (2010).

34. Regan, J.F., et al. Environmental monitoring for biological threat agents using the Autonomous Pathogen Detection System with multiplexed polymerase chain reaction. Analytical Chemistry 80, $7422-7429$ (2008).

35. Sanchez, M., Probst, L., Blazevic, E., Nakao, B. \& Northrup, M.A. The microfluidic bioagent autonomous networked detector (M-BAND): an update. Fully integrated, automated, and networked field identification of airborne pathogens. 818907-818907 (2011). 